\title{
An Accurate Measurement of the Spin- Dependent Neutron-Deuteron Scattering Length
}

\author{
B. van den Brandt ${ }^{1}$, H. Glättli ${ }^{2}$, \\ H. Grießhammer ${ }^{3}$, P. Hautle ${ }^{1}$, J. Kohlbrecher ${ }^{1}$, J.A. Konter ${ }^{1}$, \\ F.M. Piegsa $^{1,4}$, J.P. Urrego-Blanco ${ }^{1,5}$, O. Zimmer ${ }^{4}$ \\ ${ }^{1}$ Paul Scherrer Institute, CH-5232 Villigen PSI, Switzerland \\ ${ }^{2}$ SPEC and LLB, CEA Saclay, F-91191 Gif-sur-Yvette Cedex, France \\ ${ }^{3}$ Centre for Nucl. Studies, Dept. Physics, George Washington Univ., Washington, DC 20052, USA \\ ${ }^{4}$ Physics Department, Technische Universität München, D-85748 Garching, Germany \\ ${ }^{5}$ Dept. Physics and Astronomy, University of Tennessee, Knoxville, TN 37996, USA
}

\begin{abstract}
We briefly report on the progress of an experiment that has the goal to directly determine the bound spin-dependent neutron-deuteron scattering length $b_{\mathrm{i}, \mathrm{d}}$ by measuring the pseudomagnetic precession of polarized cold neutrons passing through a target with polarized nuclei. A precise measurement of $b_{\mathrm{i}, \mathrm{d}}$ can be used to increase the accuracy of the only poorly known doublet neutron-deuteron scattering length $b_{2, d}$ which is a crucial input parameter for novel effective field theories. Results of exploratory measurements of $b_{\mathrm{id}}$ are shown, which illustrate the capability of the adopted experimental method.
\end{abstract}

Keywords: Few-body physics; Neutron physics; Dynamic nuclear polarization.

PACS: 21.45.+v; 25.40.Dn; 28.20.Cz; 25.10.+s

\section{INTRODUCTION}

An accurate determination of the bound incoherent neutron-deuteron scattering length $b_{\mathrm{i}, \mathrm{d}}$ provides an important contribution to the description of few-nucleon systems at low energies using novel effective field theories [1]. These theories need two well known and independent three body constants to obtain accurate predictions of nuclear processes, e.g. in the big-bang and stellar fusion. One constant, the triton binding energy, is determined very well whereas the other one, the doublet neutrondeuteron scattering length $b_{2, \mathrm{~d}}$ is at present only known with $6 \%$ accuracy. It can be obtained via a linear combination of the accurately known spin-independent neutrondeuteron scattering length $b_{\mathrm{c}, \mathrm{d}}$ and the spin-dependent one $b_{\mathrm{i}, \mathrm{d}}$ [2].

The experiment is carried out at the cold, polarized neutron beam line FUNSPIN at SINQ at the Paul Scherrer Institute in Switzerland. It employs Ramsey's technique of separated oscillating fields [3] to measure the pseudomagnetic precession of the spin of neutrons passing through a nuclear polarized sample [4,5]. This effect is a consequence of the spin-dependent neutron index of refraction [6]. The precession angle is proportional to the bound incoherent scattering length $b_{\mathrm{i}, \mathrm{d}}$ of the nuclear species. In a sample with the thickness $d$, with various nuclear species $k$ the total pseudomagnetic precession angle $f^{*}$ is given by

CP915, Proceedings of the $17^{\text {th }}$ International Spin Physics Symposium, edited by K. Imai, T. Murakami, N. Saito, and K. Tanida 2007 American Institute of Physics 978-0-7354-0423-6/07/\$23.00 


$$
\varphi *[\mathrm{rad}]=2 \lambda d \sum_{k} \sqrt{\frac{I_{k}}{I_{k}+1}} P_{k} N_{k} b_{i, k},
$$

where? is the neutron wavelength and $I_{k}$ is the spin of the $k$-th nuclear species with the polarization $P_{k}$ and the number density $N_{k}$. To avoid absolute calibrations of the nuclear polarizations and exact measurements of ?, $d$ and $N_{k}$, this experiment measures the pseudomagnetic effect due to the deuterons relative to the very well known effect due to the protons in a single isotopically mixed target. By means of radio-frequency pulses the protons or the deuterons can be selectively depolarized to determine the corresponding precession angle [2].

\section{MEASUREMENTS AND RESULTS}

The previously observed damping of the Ramsey oscillation pattern [5] could be largely related to target polarization inhomogeneities and was cured by modifying the sample preparation process. Further, a large variety of samples has been investigated to optimise the degree of deuteration and the amount of radicaldoping.

The measurements reported below have been performed with a $1.2 \mathrm{~mm}$ thick $97 \%$ deuterated polystyrene sample, doped with a concentration of about $2 \times 10^{19} \mathrm{e}^{-} / \mathrm{cm}^{3}$ deuterated TEMPO free radical. Two Q-meter systems operating at around $16 \mathrm{MHz}$ and $106 \mathrm{MHz}$ respectively were used to measure the deuteron and proton NMR signals at the nominal field of $2.5 \mathrm{~T}$. These signals, taken as integrals over the rf-absorption lines, are proportional to $\mathrm{c}_{k} P_{k} N_{k}$, where $c_{k}$ is a factor accounting for the sensitivity of the resonance circuit. The $16 \mathrm{MHz}$ NMR circuit is a non resonant cable configuration with the tuning elements mounted inside the cryostat in close vicinity to the target. The proton NMR coil however is connected via a transmission line cable of length ? $\mathrm{rf}$ to the tuning elements situated at room temperature [7]. Figure 1 (left) shows the sample with the integrated proton NMR coil on which, at right angle (horizontally), the 7 turn deuteron coil is tightly wound (not shown). The sample is then fitted into the holder (figure 1, right) sitting in a cell filled with liquid ${ }^{4} \mathrm{He}$ that is thermally anchored to the mixing chamber of a ${ }^{3} \mathrm{He}-{ }^{4} \mathrm{He}$ dilution refrigerator [5].

For the determination of $b_{\mathrm{i}, \mathrm{d}}$ several measurements of precession angles $f$ in combination with the corresponding NMR signal integrals have to be performed (for details see [2]): First, an instrumental phase $f_{0}$ with unpolarized sample is measured before the sample is polarized via dynamic nuclear polarization DNP by which both isotopes are polarized simultaneously. After freezing the nuclear polarization one determines the "total" precession angle. Using rf-saturation, the protons are then selectively depolarized without significantly affecting the deuteron polarization and a next precession angle measurement is performed. After a subsequent depolarization of the deuterons, the measurement cycle is completed. At each step, before and after the measurement of a precession angle the deuteron and proton NMR signal integrals are recorded. To make full use of the method of relative measurement, all NMR signals have to be recorded with the same NMR system so that the instrumental factor $\mathrm{c}_{k}$ cancels, since it would be difficult to determine $\mathrm{c}_{k}$ absolutely to the required accuracy. 
This requires that the proton NMR signal has to be recorded with the deuteron NMR system at a frequency of $16 \mathrm{MHz}$. Due to the largely different gyromagnetic ratios this needs a magnetic field of about $0.4 \mathrm{~T}$. To avoid sweeping the field within a precession measurement cycle, the NMR systems have been cross calibrated; for many different polarizations the proton signal integral values at low and high field were systematically compared and an exact ratio $c_{106} / \mathrm{c}_{16}$ between the NMR systems was established. In this way we also have a much better control of the errors resulting from polarization relaxation during the field sweep, which outweighs by far the introduction of the small additional error from another NMR signal measurement.
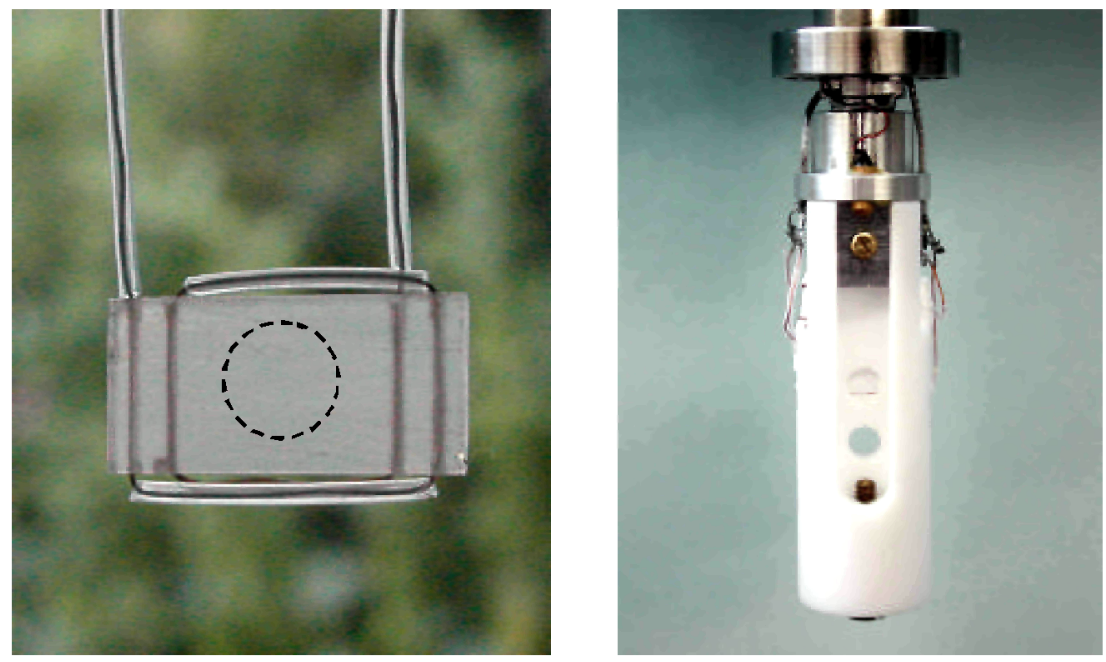

FIGURE 1. Left: Target sample slab $(7 \times 14 \times 1.2 \mathrm{~mm})$ of $97 \%$ deuterated polystyrene with the integrated $106 \mathrm{MHz}$ NMR coil. The circle indicates the beam spot defined by a $4 \mathrm{~mm}$ diameter LiF diaphragm. Right: Teflon sample holder with the sample placed behind the upper $4 \mathrm{~mm}$ diameter diaphragm. A reference neutron beam can pass through the lower opening.

During a test experiment a first series of measurements of the bound incoherent neutron deuteron scattering length has been performed. The values for different degrees of deuteron polarization are given in figure 2 and are compared to the literature value of $4.033 \pm 0.032 \mathrm{fm}$ [8]. Obviously the error bars of the measurements are not competitive yet, but the values presented here are meant to illustrate that $b_{\mathrm{i}, \mathrm{d}}$ can be determined with pseudomagnetic precession, a technique completely different from the one employed in previous measurements [8]. The error is conservatively estimated to be about $6 \%$, where the phase determination contributes with only $0.5 \%$. Distortions of the NMR signals caused difficulties to precisely determine the protondeuteron signal integral ratio and they also reduced the cross calibration accuracy. They have been caused by a short section in the transmission lines that leads from the cryostat isolation vacuum into the ${ }^{4} \mathrm{He}$ cell connecting the NMR coils. The coaxial cables employed were not well impedance matched and had a too high damping. Further, the flawed values for small deuteron polarizations point to a potential insufficiency of the NMR coil configuration used: the region of polarization sampling 
is not restricted to the beam interaction region (see figure 1, left). Polarization inhomogeneities that predominantly appear at low polarizations, e.g. at the beginning of the DNP process, can lead to wrong polarization estimations.

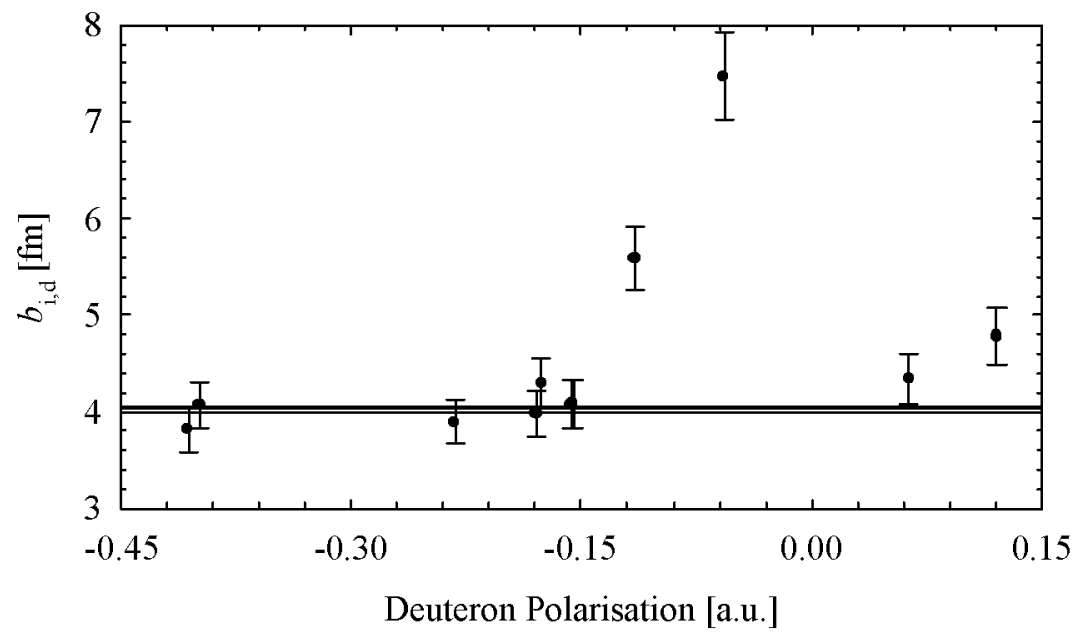

FIGURE 2. Preliminary test measurements of the bound incoherent neutron-deuteron scattering length $b_{\mathrm{i}, \mathrm{d}}$ at various target polarizations. The error bars of the measured points do not include effects due to polarization inhomogeneities of the target. The three lines give the literature value with error bars [8] for comparison.

Meanwhile the transmission lines have been replaced, the target shape reduced to exactly match the beam spot size and the NMR coil configuration correspondingly adapted. An additional saddle coil for rf-saturation has been integrated as well. With these modifications, we are confident to be able to substantially increase the accuracy for $b_{\mathrm{i}, \mathrm{d}}$ in the next stage of the experiment.

\section{REFERENCES}

1. H.W. Grießhammer, Nucl. Phys. A744, 192 (2004).

2. B. van den Brandt, H. Glättli, H.W. Grießhammer, P. Hautle, J. Kohlbrecher, J.A. Konter, O. Zimmer, Nucl. Instr. Meth. A526, 91 (2004).

3. N. Ramsey, Molecular Beams (Oxford University Press, Oxford, 1956).

4. B. van den Brandt, P. Hautle, J. Kohlbrecher, J.A. Konter, A. Michels, H. Glättli, H.W. Grießhammer, F. Piegsa, O. Zimmer, Proc. $16^{\text {th }}$ Int. Spin Physics Symposium, Trieste, Italy, 2004, pp. 669, Eds. F. Bradamante et al. (World Scientific, Singapore, 2005).

5. B. van den Brandt, H. Glättli, H.W. Grießhammer, P. Hautle, J. Kohlbrecher, J.A. Konter, F.M. Piegsa, B.S. Schlimme, J.P. Urrego-Blanco, O. Zimmer, AIP Proc. 842, 814 (2006).

6. A. Abragam, M. Goldman, Nuclear magnetism: order and disorder (Clarendon Press, Oxford, 1982).

7. P. Hautle, Nucl. Instr. Meth. A526, 76 (2004).

8. W. Dilg, L. Koester, W. Nistler, Phys. Lett. 36, 208 (1971). 